\title{
COVID-19, ФОКУС НА КАЛЬЦИЙ-ФОСФОРНЫЙ ОБМЕН
}

\author{
Маганева И.С., АйнетдиноваА.Р., Еремкина А.К., Мокрышева Н.Г.
} ФГБУ «НМИЦ эндокринологии» Минздрава России, Москва

Появляется все больше данных об ассоциации кальциево-фосфорного обмена с течением новой коронавирусной инфекции. Гипокальциемия является одним из наиболее частых биохимических нарушений и связана с тяжестью заболевания, высокой вероятностью госпитализации, необходимостью искусственной вентиляции легких и плохим прогнозом при COVID-19.

ЦЕЛЬ: исследовать основные показатели кальций-фосфорного обмена у пациентов с COVID-19 и оценить их взаимосвязи с различной степенью тяжести заболевания.

МАТЕРИАЛЫ И МЕТОДЫ: одноцентровое исследование с включением 90 пациентов (в возрасте $\geq 18$ лет) с клинически или лабораторно подтвержденной новой коронавирусной инфекцией (COVID-19). Исходные биохимические показатели, включая сывороточный кальций, фосфор, альбумин, 25 (OH) D, паратгормон, маркеры воспалительного процесса, а также инструментальная оценка тяжести COVID-19, были проведены до начала специфической иммунотерапии. Статистический анализ проводился с помощью программы Statistica 13 («StatSoft», США). Значение p<0,05 считалось статистически значимым.

PЕЗУЛЬтАТЫ: распространенность гипокальциемии (для альбумин-скорректированного кальция, ACK) $<2,15$ ммоль/л) и дефицита витамина D (<20 нг/мл) составила 36,7 и 86,7\% соответственно. Более низкое насыщение крови кислородом (SpO2) наблюдалось у пациентов с самыми низкими уровнями для АCK $(p=0,002)$ и 25(OH)D ( $p=0,001)$. Кроме того, более низкий АСК был связан с более низкими значениями SpO2 (ОШ 12 725,213, 95\% ДИ 2,661-60863,931,6), и наоборот, ACK >2,17 ммоль/л был связан с SpO2>93\% с вероятностью 71-92\%. Нами не было выявлено связи между общим и ACK, и 25(OH) D (p1=0,061 и p2=0,339 соответственно). У пациентов с тяжелой пневмонией COVID-19 (KT 3-4 степени) по сравнению с пациентами с меньшей степенью поражения (КТ 1-2 степени) отмечались значимо более низкие концентрации Са и альбумина ( $<<0,001$ для всех). Также, у пациентов с высокими уровнями СРБ (>60 мг/л) были отмечены статистически значимые различия по уровню фосфора $(1,05[0,94 ; 1,15]$ ммоль/л против $0,9[0,83 ; 0,99]$ ммоль/л; $\mathrm{p}=0,001)$. Статистическая тенденция к обратной связи была выявлена между концентрацией калия в крови и воспалительными маркерами: ферритином ( $r=-0,31 ; \mathrm{p}=0,01589)$, D-димером $(r=-0,29 ; p=0,02442), C P Б(r=-0,32 ; p=0,01220)$. Аналогичная взаимосвязь с данными параметрами выявлена при оценке сывороточного фосфора: для ферритина показатели статистической тенденции - $r=-0,32$; $\mathrm{p}=0,01168$; для СРБ - r=-0,395; $\mathrm{p}=0,00178$; для IL-6 - r=-0,26; $\mathrm{p}=0,04644$. Пациенты c IL-6 10 пг/мл или более имеют значительные различия в концентрации общего кальция $(2,25[2,18 ; 2,38]$ ммоль/л против $2,15$ [2,07; 2,23] ммоль/л; $\mathrm{p}<0,001)$. Кроме того, наблюдались тенденции к положительной корреляции АСК с уровнем лейкоцитов ( $r=0,31 ; p=0,017)$, тромбоцитов $(r=0,34 ; p=0,007)$, а также $\mathrm{SpO} 2(r=0,30 ; p=0,01928)$, c D-димером ( $r=0,33 ; p=0,00997)$, ПТГ с MHO ( $r=0,318 ; p=0,019)$, ПТГ с протромбиновым временем ( $r=0,315$; $\mathrm{p}=0,020)$ и обратная умеренная корреляция ПТГ с протромбином ( $r=-0,271 ; p=0,047)$. Регрессионный анализ показал связь высоких значений СРБ с низкими уровнями АЧТВ: $p=0,049$; отношение шансов (ОШ) 0,900, 95\% доверительный интервал (ДИ) 0,810-1,000.

Выводы: гипокальциемия и дефицит витамина D широко распространены у пациентов с тяжелым течением COVID-19, нуждающихся в госпитализации. Необходимы дальнейшие исследования для изучения влияния нарушения кальций-фосфорного обмена на COVID-19 для разработки оптимальных терапевтических стратегий. 